\title{
Registro da família Eiconaxiidae Sakai \& Ohta, 2005 (Crustacea: Decapoda: Thalassinidea) no Brasil, com redescrição de Eiconaxius carinatus (Bouvier 1925)
}

\author{
Marilena Ramos-Porto ${ }^{1,6}$, Petrônio Alves Coelho ${ }^{2,3}$, Kátia Cristina de Araújo Silva, \\ Girlene Fábia Segundo Viana ${ }^{5}$ \& Israel Hidenburgo Aniceto Cintra ${ }^{4}$ \\ ${ }^{1}$ Departamento de Pesca e Aqüicultura, \\ Universidade Federal Rural de Pernambuco - UFPE, \\ Av. Dom Manuel de Medeiros, s/n, Dois Irmãos, CEP 52171-030, Recife, PE, Brasil \\ ${ }^{2}$ Departamento de Oceanografia, \\ Universidade Federal de Pernambuco - UFPE, \\ Av. Arquitetura, s/n, Cidade Universitária, CEP 50601-910, Recife, PE, Brasil, www.ufpe.br/docean \\ ${ }^{3}$ Fundação de Amparo a Pesquisa de Estado de Alagoas - FAPEAL/CNPq, \\ e-mail: pacoelhoi@yahoo.com.br \\ ${ }^{4}$ Instituto Sócio-Ambiental e dos Recursos Hídricos, \\ Universidade Federal Rural da Amazônia - UFRA, \\ Av. Tancredo Neves, 2501, Montese, Belém, PA, Brasil, e-mail: katia.silva@ufra.edu.br \\ ${ }^{5}$ Unidade Acadêmica de Serra Talhada - UAST, \\ Universidade Federal Rural de Pernambuco - UFPE, \\ Fazenda Saco, s/n, CP 063, CEP 56900-000, Serra Talhada, PE, Brasil, e-mail: fabiaviana@yahoo.com.br \\ ${ }^{6}$ Autor para correspondência: Marilena Ramos-Porto, e-mail: lenaporto@gmail.com
}

RAMOS-PORTO, M., COELHO, P.A., SILVA, K.C.A., VIANA, G.F.S. \& CINTRA, I.H.A. 2008. First record of family Eiconaxiidae Sakai \& Ohta, 2005 (Crustacea: Decapoda: Thalassinidea) in Brazil, with redescription of Eiconaxius carinatus (Bouvier 1925). Biota Neotrop. 8(3): http://www.biotaneotropica.org.br/v8n3/en/ abstract?article+bn00908032008.

Abstract: Eiconaxius carinatus (Bouvier 1925) is characterized, mostly, by smooth rostral lateral carinae, merus of major chela with smooth dorsal carina, palm of major chela with dorsal carina evident and length of fingers of minor chela less than the palm length. One specimen was collected off the state of Maranhão $\left(00^{\circ} 08^{\prime} \mathrm{N}\right.$ and $44^{\circ} 32^{\prime} \mathrm{W}$ ) in a depth of $247 \mathrm{~m}$; this species was known previously from Florida and West Indies (Saint Croix and Saint Vincent), in depths between 161 and $339 \mathrm{~m}$. This record is the first record of this family for Brazil. Keywords: Decapoda, Thalassinidea, Eiconaxiidae, Eiconaxius carinatus, first record, Brazil.

RAMOS-PORTO, M., COELHO, P.A., SILVA, K.C.A., VIANA, G.F.S. \& CINTRA, I.H.A. 2008. Primeiro registro da família Eiconaxiidae Sakai \& Ohta, 2005 (Crustacea: Decapoda: Thalassinidea) no Brasil, com redescrição de Eiconaxius carinatus (Bouvier 1925). Biota Neotrop. 8(3): http://www.biotaneotropica.org.br/ v8n3/pt/abstract?article+bn00908032008.

Resumo: Eiconaxius carinatus (Bouvier 1925) caracteriza-se, principalmente, por apresentar carenas laterais do rostro lisas, mero da quela maior com carena dorsal lisa, palma da quela maior exibindo carena dorsal bem nítida e dedos da quela menor de comprimento inferior ao da palma. Um exemplar foi coletado ao largo do Estado do Maranhão $\left(00^{\circ} 08^{\prime} \mathrm{N}\right.$ e $\left.44^{\circ} 32^{\prime} \mathrm{W}\right)$ a $247 \mathrm{~m}$ de profundidade. A área de ocorrência anteriormente conhecida abrangia a Flórida e Antilhas (Santa Cruz e São Vicente), entre 161 e 339 m. Este trabalho registra pela primeira vez a família para o Brasil.

Palavras-chave: Decapoda, Thalassinidea, Eiconaxiidae, Eiconaxius carinatus, primeiro registro, Brasil. 


\section{Introdução}

Os Thalassinidea representam um grupo de crustáceos decápodos bem sucedidos. Caracterizam-se por apresentar abdome simétrico, achatado no sentido dorso-ventral, com leque caudal bem desenvolvido; os dois primeiros pares de pereiópodos são quelados, o primeiro deles o mais desenvolvido. No Brasil, as espécies são encontradas em ambiente marinho ou salobro, fazendo parte da fauna endopsâmica; ocorrem em ambientes costeiros, porém algumas estão registradas em águas mais profundas, o que dificulta o conhecimento destes animais. De acordo com Martin \& Davis (2001) e Sakai \& Ohta (2005), o grupo compreende 12 famílias distribuídas em três superfamílias (Axioidea, Callianassoidea e Thalassinoidea).

A família Eiconaxiidae Sakai \& Ohta, 2005, incluída na superfamília Axioidea Huxley, 1879, foi erigida para abrigar apenas o gênero Eiconaxius Bate, 1888, o qual, inicialmente, continha três espécies, E. acutifrons Bate, 1888, E. kermadeci Bate, 1888 e E. parvus Bate, 1888, a primeira encontrada no Mar de Célebes e as demais perto das Ilhas Kermadec. Recentemente, Sakai \& Ohta (2005) incluíram 27 espécies no gênero, encontradas em águas tropicais ou subtropicais em várias partes do mundo, porém nenhuma citada para o litoral brasileiro. O presente trabalho tem por objetivo registrar a presença desta família no Brasil.

\section{Material e Métodos}

O material examinado foi coletado durante prospecções oceanográficas realizadas pelo Navio de Pesquisa "Almirante Paulo Moreira”, pertencente ao Centro de Pesquisas e Gestão de Recursos Pesqueiros do Litoral Norte (CEPNOR/IBAMA-MMA), durante o Programa de Avaliação do Potencial Sustentável dos Recursos Vivos da Zona Econômica Exclusiva - Programa REVIZEE, Subcomitê Regional de Pesquisa Costa Norte (REVIZEE, Score Norte). As capturas foram efetuadas através de arrastos com rede comercial para camarões, com duração de 30 minutos a 1 hora. A bordo, o material biológico foi acondicionado em bandejas e colocado em câmara frigorífica. O exemplar foi medido do rostro ao telson (em milímetros) e identificado no Laboratório de Carcinologia do Departamento de Oceanografia da Universidade Federal de Pernambuco (DOPE) e encontra-se depositado na Coleção Carcinológica do referido departamento. A redescrição está embasada no espécime examinado e na literatura consultada.

\section{Resultados}

Superfamilia AXIOIDEA Huxley, 1879

Diagnose - Rostro desenvolvido. Sulco cervical distinto ou indistinto. Linha talassínica ausente. Pedúnculos oculares cilíndricos ou ovais. Escafognatito com flagelo posterior. Primeiros pereiópodos quelados, iguais ou desiguais; segundos quelados; terceiros simples; quartos e quintos simples ou subquelados, ou, ainda, terceiro a quinto pares com dáctilo oval. Primeiros pleópodos presentes ou ausentes; segundos birremes, de forma semelhante aos demais, com apêndice interno no endopodito (adaptado de Sakai \& Ohta 2005).

Famílias AXIIDAE Huxley, 1879; CALOCARIDIDAE Ortmann, 1891; EICONAXIIDAE Sakai \& Ohta, 2005, MICHELEIDAE Sakai, 1992 e STRAHLAXIIDAE Poore, 1994

Família EICONAXIIDAE Sakai \& Ohta, 2005

Diagnose - Rostro triangular, ultrapassando os pedúnculos oculares, extremidade arredondada, superfície dorsal côncava. Carapaça com carenas submedianas em forma de ferradura, sem tubérculos; sulco cervical indistinto; linha talassínica ausente. Pedúnculos oculares fortes; olhos pigmentados; espinhos supraoculares ausentes. Escafocerito moderadamente longo. Pereiópodos do primeiro par robustos, com forma e tamanho diferentes em ambos os sexos; dáctilo e propódio com as margens externas sem espinhos ou tubérculos. Pereiópodos do terceiro ao quinto pares com dáctilo simples, arredondado; margem ventral com espínulos. Machos com primeiro par de pleópodos ausentes; demais pares estreitos e birremes, todos com apêndice interno; apêndice masculino nos pleópodos do segundo par. Fêmeas com primeiros pleópodos unirremes; os demais estreitos, birremes, com apêndice interno. Exopodito do urópodo sem sutura (modificado de Sakai \& Ohta 2005). Compreende um único gênero: Eiconaxius Bate, 1888.

Gênero Eiconaxius Bate, 1888

Eiconaxius Bate, 1888: 40. Sakai \& Saint Laurent, 1989: 2, 15. Sakai \& Ohta, 2005:69

Iconaxiopsis Alcock, 1901: 193

Axius (Eiconaxius) - De Man, 1925:4, 10. Balss, 1957:1579

Espécie tipo - Eiconaxius acutifrons Bate, 1888

Espécies válidas conhecidas - Eiconaxius acutifrons Bate, 1888; E. agassizi Bouvier, 1905; E. albatrossae Kensley, 1996; E. andamanensis (Alcock 1901); E. antillensis Bouvier, 1905; E. asper Rathbun, 1906; E. baja Kensley, 1996; E. borradailei Bouvier, 1905; E. caribbaeus (Faxon 1896); E. carinatus Bouvier, 1925; E. consobrinus (De Man 1907); E. cristagalli (Faxon 1893); E. demani Sakai, 1992; E. farreae Ortmann 1891; E. hakuhou Sakai \& Ohta, 2005; E. indicus (De Man 1907); E. kermadecensis (Chilton 1911); E. kermadeci Bate, 1888; E. kimbla Kensley, 1996; E. laccadivensis (Alcock \& Anderson 1894); E. mortenseni Sakai, 1992; E. parvus Bate, 1888; E. rotundifrons Bouvier, 1905; E. sibogae De Man 1925; E. singularis Zarenkov, 1981; E. spinigera (MacGilchrist 1905) e E. weberi (De Man 1905), de acordo com Sakai \& Ohta (2005) e Bisby et al. (2006; 2007).

Eiconaxius carinatus (Bouvier 1925) (Figuras 1-10)

Axius (Eiconaxius) caribbaeus carinatus Bouvier, 1925:465, pl. 9, Figura 3 - Schmitt, 1935:191, Figure 52

Eiconaxius carinatus - Sakai \& Saint Laurent, 1989:2

Localidade tipo - Santa Cruz: "Blake" estação 139, profundidade 218 braças $(398,5 \mathrm{~m})$.

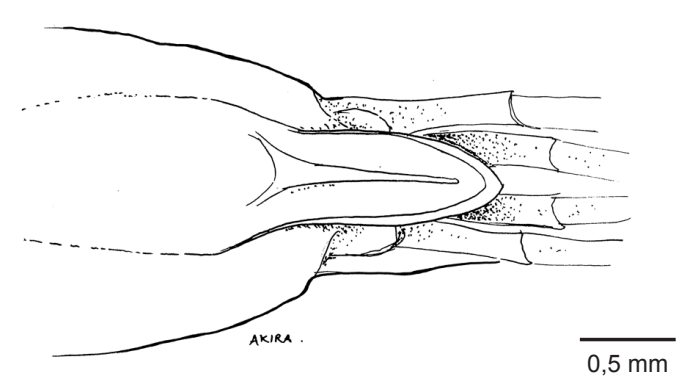

Figura 1. Eiconaxius carinatus (Bouvier, 1925); rostro, vista dorsal.

Figure 1. Eiconaxius carinatus (Bouvier, 1925); rostrum, dorsal view. 
Outros registros - Milligan Key, Flórida: “Blake”, estação 259, profundidade 124 braças (227 m); São Vicente: "Blake" estação 232, profundidade 88 braças (161 m) (Bouvier, 1925).

Material - Maranhão: REVIZEE-Score Norte, Prospecção 22 , estação $15,00^{\circ} 08^{\prime} \mathrm{N}$ e $44^{\circ} 32^{\prime} \mathrm{W}, 247 \mathrm{~m}$ de profundidade, 1 macho $(15,0 \mathrm{~mm})\left(\mathrm{DOPE} \mathrm{n}^{\circ} 13.417\right)$.

Descrição - Carapaça lisa. Região gástrica com carena mediana não alcançando a extremidade do rostro, ramificando-se posteriormente em duas carenas sub-laterais distintas; carenas laterais lisas, prolongando-se a partir da carena marginal do rostro. Sulco cervical pouco distinto. Rostro lanceolado, margens laterais e carena mediana lisas; extremidade arredondada, ultrapassando a porção distal do artículo basal do pedúnculo antenular (Figuras 1, 2 e 3). Pedúnculos oculares espessos e curtos, ligeiramente menores que a metade do comprimento do rostro; córnea ocupando a maior parte do pedúnculo ocular e levemente pigmentada. Escafoceritos ultrapassando os pedúnculos antenulares e quase do mesmo tamanho que os pedúnculos antenais. Maxilípedes do terceiro par com exopodito.

Primeiros pereiópodos desiguais (Figuras 4, 5). Quelípede maior (Figuras 6, 7) com coxa e base sem espinhos. Ísquio com dois dentes nas margens ventral e dorsal. Mero cerca de 1,5 vezes tão longo quanto largo, margem ventral denticulada e dorsal com carena lisa, superfície externa com carena longitudinal pouco definida. Carpo mais largo do que longo, 2/3 do comprimento

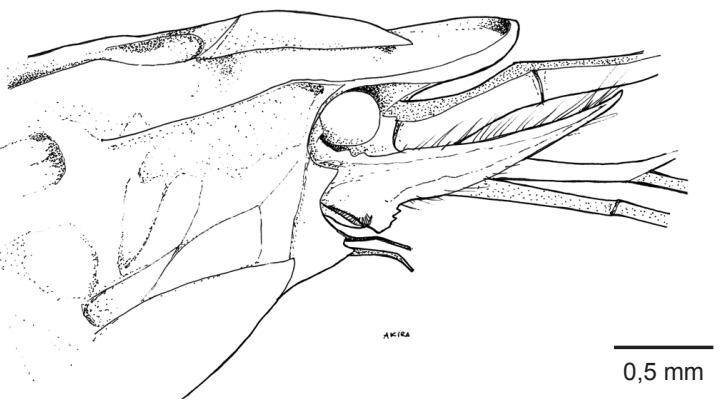

Figura 2. Eiconaxius carinatus (Bouvier, 1925); rostro, vista lateral.

Figure 2. Eiconaxius carinatus (Bouvier, 1925); rostrum, lateral view.

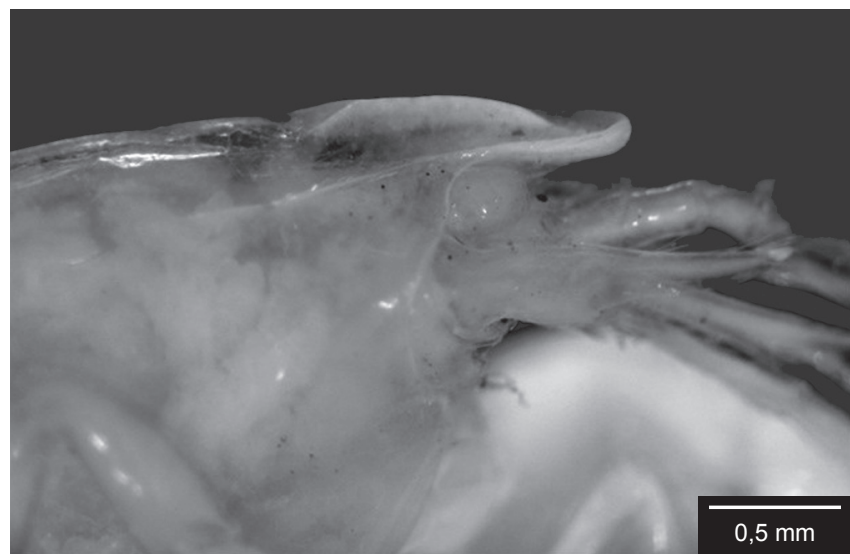

Figura 3. Eiconaxius carinatus (Bouvier, 1925); rostro, vista lateral.

Figure 3. Eiconaxius carinatus (Bouvier, 1925); rostrum, lateral view. do mero, cerca de metade do comprimento dorsal da palma; margem ventral lisa com um dente agudo subterminal. Quela pouco menos de duas vezes tão longa quanto larga; dedos com extremidades entrecruzadas, deixando um espaço interdigital bem visível; palma com alguns tubérculos redondos e tufos de cerdas, ligeiramente divergindo em altura distalmente, margem dorsal com uma carena lisa terminando em um dente agudo distal e outro menor, subdistal, margem distal convexa, com um dente obtuso no ângulo ventral; face externa com uma carena ventral, duplicada superiormente por uma linha de grânulos diminutos que se dirige para frente, em direção ao ápice do pólex; pólex côncavo proximalmente, margem cortante grosseiramente denticulada, superfícies lateral e mesial com uma concavidade, tubérculos redondos e tufos de cerdas dispersos na superfície externa; dáctilo carenado ao longo da margem dorsal, margem cortante ligeiramente denticulada, com dois dentes proximais e três tubérculos redondos na face externa, próximos à sua base.

Quelípede menor (Figura 8) com coxa e base sem espinhos. Ísquio com alguns dentes irregulares e uma carena dorsal com três espínulos. Mero tão longo quanto largo, margem ventral grosseiramente denticulada, sem espínulos distais; margem dorsal com três dentes no terço distal. Carpo metade do comprimento do mero, margem ventral com dente subdistal. Quela pouco mais de duas vezes sua maior altura; palma cerca da metade do comprimento da quela, margem distal com um dente basal e dois

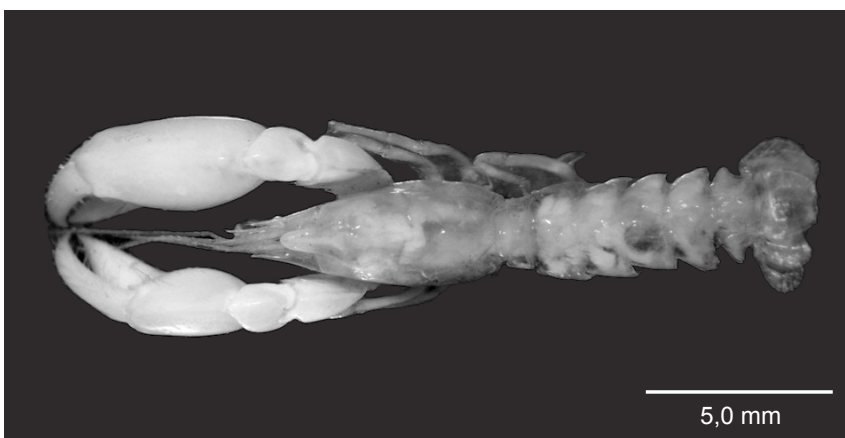

Figura 4. Eiconaxius carinatus (Bouvier, 1925); macho, vista dorsal.

Figure 4. Eiconaxius carinatus (Bouvier, 1925); male, dorsal view.

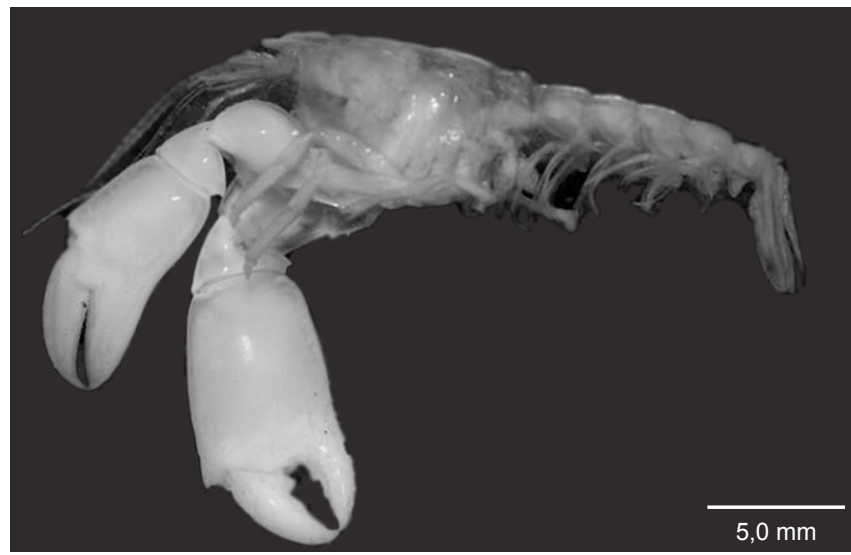

Figura 5. Eiconaxius carinatus (Bouvier, 1925); macho, vista lateral.

Figure 5. Eiconaxius carinatus (Bouvier, 1925); male, lateral view. 


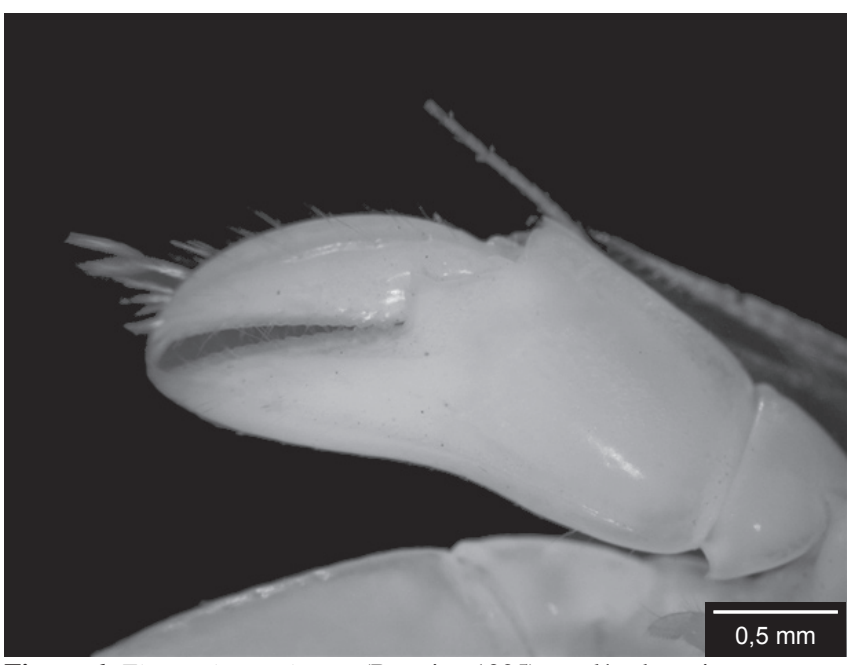

Figura 6. Eiconaxius carinatus (Bouvier, 1925); quelípede maior.

Figure 6. Eiconaxius carinatus (Bouvier, 1925); larger cheliped.

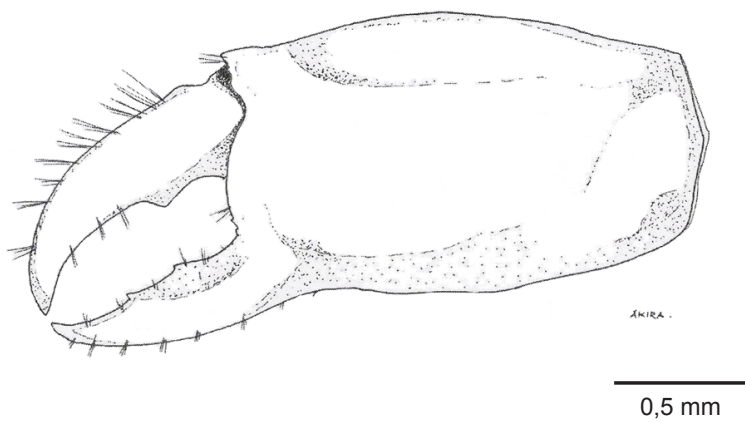

Figura 7. Eiconaxius carinatus (Bouvier, 1925); quelípede maior.

Figure 7. Eiconaxius carinatus (Bouvier, 1925); larger cheliped.

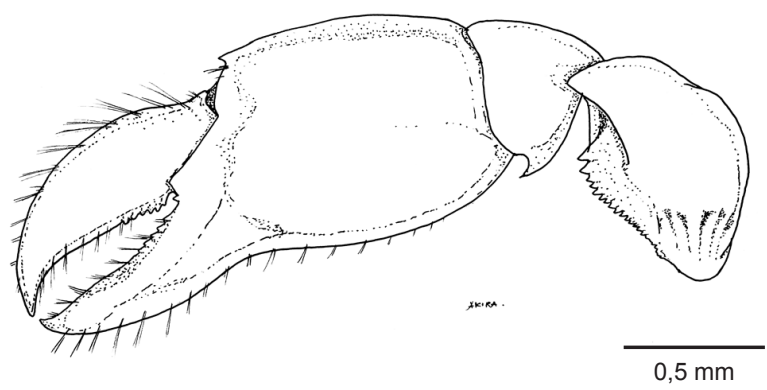

Figura 8. Eiconaxius carinatus (Bouvier, 1925); quelípede menor.

Figure 8. Eiconaxius carinatus (Bouvier, 1925); smaller cheliped.

apicais; margem dorsal com um dente distal; face externa com uma carena próxima à margem ventral que se estende em direção à extremidade do pólex, acompanhada por linha de grânulos; face interna com uma crista denticulada situada sobre as bases do pólex e do dáctilo; pólex quase igual ao comprimento dorsal da palma, margem cortante denticulada, superfície lateral com uma carena longitudinal paralela à esta margem, sem espaço interdi- gital; dáctilo cerca de 1,5 vezes o comprimento dorsal da palma, aproximadamente quatro vezes tão longo quanto largo, margem cortante denticulada, dentículos basais um pouco maiores que os restantes, duas carenas fortes e lisas, uma dorsal e uma lateral, sendo esta última interrompida próxima à base do dáctilo.

Terceiros pereiópodos simples, propódio menos de duas vezes o comprimento do carpo, com tufos de cerdas duras na margem ventral; dáctilo foliáceo, com uma fileira de espínulos na margem disto-ventral. Quartos pereiópodos com propódio menos de duas vezes o comprimento do carpo; dáctilo semelhante ao dos pereiópodos precedentes. Quintos pereiópodos com propódio mais de duas vezes o comprimento do carpo, com tufo de cerdas no ângulo ventro-distal; dáctilo semelhante ao dos pereiópodos precedentes.

Abdômen liso; primeiro somito curto, segundo ao sexto de comprimento quase igual; pleuras do segundo somito bastante defletidas posteriormente, em ângulo póstero-lateral sub-agudo; terceiro com pleuras semelhantes às do segundo; quarto com ângulo posterior das pleuras menos agudo que nos precedentes, margem posterior com um dentículo; quinto com margem póstero-lateral das pleuras convexa; sexto com entalhe na margem posterior das pleuras (Figura 9).

Pleópodos do primeiro par ausentes; pleópodos do segundo ao quinto pares birremes, delgados; endopodito com apêndice interno. Urópodos (Figura 10) com exopodito bastante convexo, margens laterais espinulosas; endopodito espinuloso nas margens distal e lateral. Telson (Figura 10) um pouco mais longo do que largo, muito mais longo que o sexto somito, com uma área mediana rebaixada; margens laterais quase paralelas, com cinco espínulos; margem posterior com cerdas.

\section{Discussão}

O gênero Axius, erigido por Leach em 1815 foi subdividido por Borradaile (1903) em cinco subgêneros que, à exceção de Neaxius erigido como subgênero naquela oportunidade, eram até então considerados como gêneros válidos. Desta forma, Axius passou a compreender os subgêneros Axius, Neaxius Borradaille, 1903, Iconaxiopsis Alcock, 1901, Eiconaxius Bate, 1888 e Paraxius Bate, 1888, embora a validade do Iconaxiopsis the parecesse duvidosa (De Man, 1925).

Estudando os Axiidae coletados pelo "Blake", Bouvier (1925) classificou a espécie Iconaxius caribbaeus Faxon, 1896 no gênero Axius e no subgênero Eiconaxius e a dividiu em três subespécies: Axius (Eiconaxius) caribbaeus (Faxon, 1896), A. (E.) caribbaeus rotundifrons Bouvier, 1905 e A. (E.) caribbaeus carinatus, esta última descrita naquela ocasião. De Man (1925), evidentemente ignorando o trabalho de Bouvier, publicado naquele mesmo ano, considerou A. (E.) caribbaeus e A. (E.) rotundifrons como espécies distintas, incluindo diagnoses de ambas e indicou as principais diferenças em relação à forma típica. Schmitt (1935) publicou diagnose e figura de Axius (Eiconaxius) caribbaeus carinatus. Quanto a Eiconaxius, trabalhos recentes vêm considerando-o como gênero válido (Sakai \& Saint Laurent 1989, McLaughlin et al. 2005, Sakai \& Ohta 2005, Bisby et al. 2007).

A descrição original de E. carinatus é muito sucinta, baseandose, principalmente, sobre caracteres do ísquio da quela maior e das pleuras abdominais. Schmitt (1935) acrescentou novas informações concernentes ao rostro, carapaça e escafocerito, além de ratificar aquelas contidas na descrição original. A presente redescrição confirma os caracteres específicos encontrados pelos autores anteriores e acrescenta muitos outros relativos à carapaça, quelípedes, pereiópodos, abdômen, pleópodos e telson. 


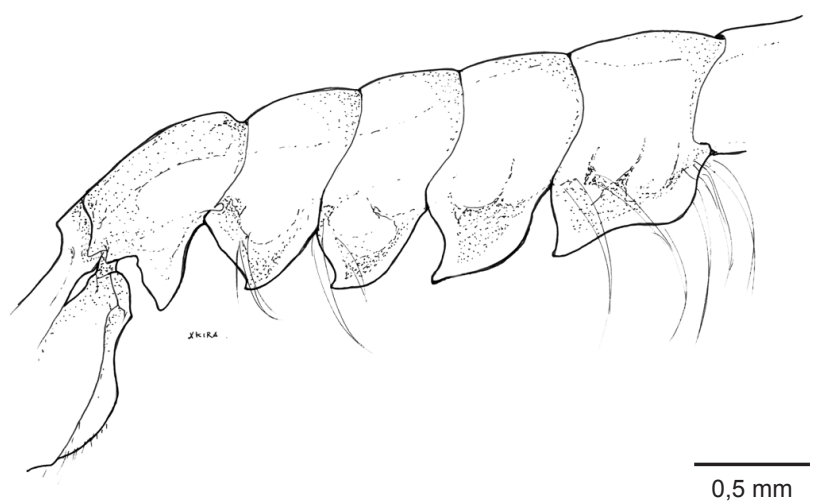

Figura 9. Eiconaxius carinatus (Bouvier 1925); abdômen, vista lateral. Comprimento do abdomen, 7,0 mm.

Figure 9. Eiconaxius carinatus (Bouvier 1925); abdomen, lateral view. Length of abdomen, $7.0 \mathrm{~mm}$.

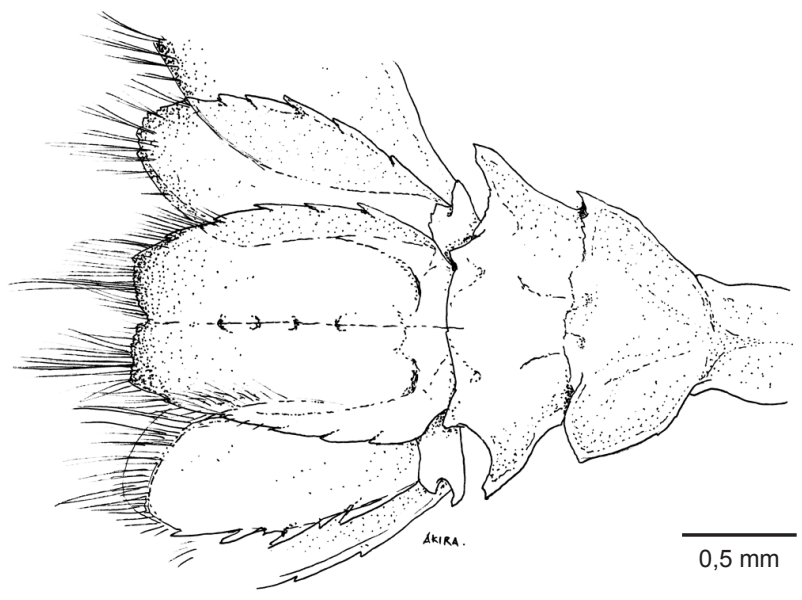

Figura 10. Eiconaxius carinatus (Bouvier, 1925); telson e endopodito dos urópodos.

Figure 10. Eiconaxius carinatus (Bouvier, 1925); telson and uropods endopodite.

O trabalho atual comprova a validade de E. carinatus, tal como aceita por Sakai \& Saint Laurent (1989) e por Bisby (2006; 2007). Com efeito, embora E. rotundifrons, E. caribbaeus e E. carinatus sejam muito semelhantes entre si, existem diferenças importantes permitindo individualizá-las. Salienta-se que a porção anterior da carapaça de $E$. carinatus é praticamente idêntica à de $E$. caribbaeus e bastante diferente daquela de E. rotundifrons, a qual apresenta carenas laterais do rostro crenuladas ou denteadas. Além disto, o mero da quela maior de E. carinatus possui a carena dorsal lisa, enquanto em E. caribbaeus esta carena é dotada de três a quatro dentículos; a palma da quela maior de E. carinatus exibe carena dorsal bem nítida, enquanto a de E. caribbaeus é desprovida desta carena; em E. carinatus os dedos da quela maior são dotados de carenas, grânulos e tubérculos e em E. caribbaeus existe apenas uma carena ventral no pólex. E. rotundifrons se assemelha à E. carinatus pelos caracteres das pleuras do abdômen, urópodos e telson, porém difere pela porção dorsal do pólex da quela maior, onde os dentes encontrados em E. carinatus são substituídos por uma carena dentiforme. Finalmente, os dedos da quela menor de E. rotundifrons e de E. caribbaeus são cerca de duas vezes o comprimento da palma, enquanto os de $E$. carinatus são menores que a palma.

Considerando as diferenças observadas, os autores são da opinião que $E$. carinatus deve ser mantida como espécie válida, até que o encontro de novos exemplares permita uma melhor avaliação das diferenças observadas entre ela, E. rotundifrons e E. caribbaeus.

\section{Agradecimentos}

Os autores expressam seus agradecimentos aos doutorandos Jesser Fidelis e Eduardo Esteves Leal (UFRJ) e Luis Ernesto Arruda (UFPE) pela ajuda nas fotografias e ao engenheiro de pesca Almir Akira Inada pela confecção dos desenhos.

\section{Referências Bibliográficas}

BALSS, H. 1957. Decapoda. In (H.G. Bronns ed.). Klassen und Ordnungen des Tierreichs. Leipzig. Fünfter Band, I. Abteilung, 7. Buch, 12. Lieferung: VIII, Systematik: 1505-1672.

BISBY, F.A., RUGGIERO, M.A., ROSKOV, Y.R., CACHUELA-PALACIO, M., KIMANI, S.W. \& KIRK, P.M., Soulier-Perkins, A. \& Hertum, J. van. 2006. Species 2000 \& ITIS Catalogue of Life: 2006 Annual Checklist. Species 2000. Reading, U.K. (ultimo Acesso em 18/08/2006). (CDROM).

BISBY, F.A., ROSKOV, Y.R., RUGGIERO, M.A, ORRELL, T.M., PAGLINAWAN, L.E., BREWER, P.W., BAILLY, N. \& HERTUM, J. VAN. 2007. Species 2000 \& ITIS Catalogue of Life: 2007 Annual Checklist: www.catalogueoflife.org/annual-checklist/2007. (último acesso em 03/07/2007).

BOUVIER, E.L. 1925. Reports on the Results of Dredging under the supervision of Alexander Agassiz in the Gulf of Mexico, the Caribbean Sea, and along the Atlantic Coast of the United States, 1877-80, by the U.S. Coast Survey Steamer "Blake". XIVIII. Les Macroures Marcheurs. Mem. Mus. Comp. Zool. 47(5):401-472.

De MAN, J.G. 1928. The Decapoda of the Siboga-Expedition. Part 6. The Axiidae collected by the Siboga-Expedition. Siboga Expedition. Sigoba Expeditie 39a(5): 1-127.

MCLAUGHLIN, P.A., CAMP, D.K., ANGEL, M.V., BOUSFIELD, E.L., BRUNEI, P., BRUSCA, R.C., CADIEN, V., COHEN, A.C., CONLAN, K., ELDREDGE, L.G., FELDER, D.L., GOY, J.W., HANEY, T., HANN, B., HEARD, R.W., HENDRYCKS, E.A., HOBBS, H.H., HOLSINGER, J.R., KENSLEY, B., LAUBITZ, D.R., LECROY, S.E., LEMAITRE, R., MADDOCKS, R.F., MARTIN, J.W., MIKKELSEN, P., NELSON, E., NEWMAN, W. A., OVERSTREET, R. M., POLY, W.J., PRICE, W., REID, J.W., ROBERTSON, A., ROGERS, D.C., ROSS, A., SCHOTTE, M., SCHRAM, F.R., SHIH, C.T., WATLING, L., WILSON, G.D.F. \& TURGEON, D.D. 2005. Common and scientific names of aquatic invertebrates from the United States and Canada: crustaceans. American Fisheries Society, Bethesda, Maryland. (Special Publication 31).

MARTIN, J.W. \& DAVIS, G.E. 2001. An updated classification of the recent Crustacea. Natural History Museum of Los Angeles County. 132p. (Science Series 39).

SAKAI, K. \& OHTA, S. 2005. Some Thalassinid Collections By R/V "Hakuhou-Maru" and R/V "Tansei-Maru", University of Tokyo, in the Sulu Sea, Philippines, and in Sagami Bay and Suruga Bay, Japan, including two new species, one new genus, and one new family (Decapoda, Thalassinidea). Crustaceana. 78(1):67-93.

SAKAI, K. \& DE SAINT-LAURENT, M. 1989. A check list of Axiidae (Decapoda, Crustacea, Thalassinidea, Anomura), with remarks and in addition descriptions of one new subfamily, eleven new genera and two new species. Naturalists 3: 1-104.

SCHMITT, W.L. 1935. Crustacea Macrura and Anomura of Porto Rico and the Virgin Islands. Sci. Surv. Puerto Rico and Virgin Islands 159(2):125-227. 